\title{
LASER PULSE VARIATIONS AND THEIR INFLUENCE ON RADIOMETRIC CALIBRATION OF FULL-WAVEFORM LASER SCANNER DATA
}

\author{
A. Roncat ${ }^{\mathrm{a}, *}$, H. Lehner ${ }^{\mathrm{a}}$, C. Briese $\mathrm{a}^{\mathrm{a}, \mathrm{b}}$ \\ ${ }^{\text {a }}$ Institute of Photogrammetry and Remote Sensing (IPF), Vienna University of Technology, Austria - \\ (ar, hl, cb)@ipf .tuwien.ac.at, www.ipf .tuwien.ac.at \\ ${ }^{\mathrm{b}}$ Ludwig Boltzmann Institute for Archaeological Prospection and Virtual Archaeology, Vienna, Austria - \\ christian. briese@archpro.lbg.ac.at, archpro.lbg.ac.at
}

Commission VII - WG VII/7

KEY WORDS: Radiometric Calibration, Full-Waveform, Laser Scanning, Error Assessment

\begin{abstract}
:
Full-waveform laser scanning extends the information content of "conventional" laser scanning by storing the temporal profile of both the emitted laser pulse and its echoes. This allows for calculating radiometric quantities in addition to the geometric data. This radiometric information needs to be calibrated in order to enable comparison among flight strips of the same laser scanner campaign and/or different campaigns. Radiometric calibration is aimed at the determination of a calibration constant which contains the parameters of the emitted laser pulse (besides others). All of these parameters are normally treated as constants. In this paper, the sensitivity of the calibration constant to variations of the emitted laser pulse is analysed theoretically by deriving it according to the error propagation law, followed by an empirical analysis carried out on the example of two airborne full-waveform laser scanning campaigns. Both were operated with the same instrument and over the same area on two different dates.
\end{abstract}

\section{INTRODUCTION}

Airborne laser scanning (ALS) has become a standard technique for the acquisition of three-dimensional topographic data during the last 15 years. Besides geometric data, i.e. the point cloud, current instruments record also radiometric information. This might be an integer number, commonly referred to as intensity, stored as additional attribute of the single points. Moreover, a sampled copy of the temporal shape of the emitted laser pulse and of its echoes can be recorded. This is referred to as full-waveform laser scanning (Mallet and Bretar, 2009).

Radiometric data needs to be calibrated if its information content is to be compared among flight strips of different altitude or flight campaigns carried out at different dates and/or with different instruments (Höfle and Pfeifer, 2007). The physical basis for the calibration is the radar equation (Jelalian, 1992), the sought quantity is the calibration constant. Its calculation is presented in detail in Section 2.

This study focuses on quantifying the uncertainty of the calibration constant caused by variations of the emitted laser pulse. The corresponding derivations are presented in Section 3. The subsequent section contains an empirical analysis w.r.t. to these variations carried out on behalf of multi-temporal data sets. These data were acquired over the same area in 2004 and 2005. The results are presented in Section 5 and the conclusions are given in Section 6.

\section{THEORY}

\subsection{Radar Equation and Backscatter Cross-Section}

The relation of the transmitted laser power $P_{t}(t)$ and the detected power of its echo $P_{d}(t)$ is given by the radar equation (Jelalian,

\footnotetext{
${ }^{*}$ Corresponding author.
}

1992):

$$
P_{d}(t)=\frac{D_{r}^{2}}{4 \pi R^{4} \beta_{t}^{2}} P_{t}\left(t-\frac{2 R}{v_{g}}\right) \sigma \eta_{\mathrm{SYS}} \eta_{\mathrm{ATM}}
$$

with $\beta_{t}$ denoting the beamwidth of the transmitted signal, $R$ the range from the sensor to the target, $t$ the travel time, $v_{g}$ the group velocity of the laser ray, $\sigma$ the effective backscatter cross-section (in $\mathrm{m}^{2}$ ), $D_{r}$ the aperture diameter, $\eta_{\mathrm{ATM}}$ the atmospheric transmission factor, and $\eta_{\mathrm{SYS}}$ the system transmission factor. The backscatter cross-section is a product of the target area $\left(\mathrm{d} A\left[\mathrm{~m}^{2}\right]\right)$, the target reflectivity $(\varrho[])$, and the factor $4 \pi / \Omega$ describing the scattering angle of the target $(\Omega[\mathrm{sr}])$ in relation to an isotropic scatterer (Jelalian, 1992):

$$
\sigma=\frac{4 \pi}{\Omega} \varrho \mathrm{d} A
$$

$P_{d}(t)$ is digitized in intervals of normally $1 \mathrm{~ns}$ (Mallet and Bretar, 2009). Since the range resolution is limited by the digitization interval and the width of the emitted laser pulse, typically scatterers closer than $0.5 \mathrm{~m}$ to each other can therefore not be separated. This results in the aggregation of such scatterers, giving the radar equation the following form (Wagner et al., 2006):

$$
P_{d}(t)=\sum_{i=1}^{N} \frac{D_{r}^{2}}{4 \pi R_{i}^{4} \beta_{t}^{2}} \eta_{\mathrm{SYS}} \eta_{\mathrm{ATM}} P_{t}(t) \otimes \sigma_{i}^{\prime}(t)
$$

with $\sigma_{i}^{\prime}(t):=\partial \sigma_{i} / \partial t$ as the differential backscatter cross-section and $\otimes$ as the convolution operator.

In fact, not $P_{d}(t)$ is recorded but its convolution with the impulse response of the receiver $\Gamma(t)$ :

$$
P_{r}(t):=P_{d}(t) \otimes \Gamma(t)
$$

This leads to a term $P_{t}(t) \otimes \sigma_{i}^{\prime}(t) \otimes \Gamma(t)$ on the right-hand side of Equation (3). Convolution is commutative, i.e.

$$
P_{t}(t) \otimes \sigma_{i}^{\prime}(t) \otimes \Gamma(t)=P_{t}(t) \otimes \Gamma(t) \otimes \sigma_{i}^{\prime}(t)
$$


The first two factors on the right-hand side form the system waveform $S(t):=P_{t}(t) \otimes \Gamma(t)$. In the scanner, a damped copy of the emitted waveform is sent directly to the receiver so that a waveform proportional to $S(t)$ is recorded. Thus, it is possible to base our calculations on the recorded waveforms $P_{r}(t)$ and $S(t)$ instead of the (unknown) quantities $P_{d}(t)$ and $P_{t}(t)$, resp.

Extracting the differential backscatter cross-section $\sigma_{i}^{\prime}(t)$ for given $P_{r}(t)$ and $S(t)$ implies deconvolution. Several approaches for solving this task in full-waveform laser scanning have been developed, e.g.:

- Gaussian Decomposition (Hofton et al., 2000; Wagner et al., 2006) solves the deconvolution implicitly: Both $S(t)$ and $P_{r}(t)$ are modeled with Gaussian functions. Since the convolution of two Gaussians again gives a Gaussian, the derivation of $\sigma_{i}^{\prime}(t)$ is straightforward.

- The algorithm presented in (Jutzi and Stilla, 2006) comprises the transformation of the emitted pulse and the received waveform to the frequency domain. Thus, the differential backscatter cross-section is retrieved as the result of division of the spectrum of the received waveform by the spectrum of the emitted pulse. In this approach, a Wiener Filter is applied for noise reduction in the frequency domain.

- The EM (expectation-maximization) approach presented in (Parrish and Nowak, 2009) attempts to model $\sigma_{i}(t)$ as a chain of discrete spikes in time domain. The focus of this approach lies on extracting of the correct number of echoes and their exact positions rather than gaining radiometric information.

- Deconvolution based on uniform B-splines (Roncat et al., 2011) models $S(t)$ and $P_{r}(t)$ as uniform B-spline curves of different degrees. By exploiting the convolution properties of this kind of functions, deconvolution can be performed in a linear approach.

In the subsequent text, we will follow the terminology of Gaussian Decomposition. However, the results are not limited to this approach.

\subsection{Calibration Constant}

As mentioned before, $S(t)$ and $P_{r}(t)$ are both modeled as Gaussian functions:

$$
S(t)=\hat{S} e^{-\frac{\left(t-t_{s}\right)^{2}}{2 s_{s}^{2}}} \quad P_{r}(t)=\sum_{i=1}^{N} \hat{P}_{i} e^{-\frac{\left(t-t_{i}\right)^{2}}{2 s_{p, i}^{2}}}
$$

with

$$
\hat{P}_{i}=\frac{D_{r}^{2}}{4 \pi R_{i}^{4} \beta_{t}^{2}} \frac{\hat{S} s_{s}}{s_{p, i}} \sigma_{i} \eta_{\mathrm{SYS}} \eta_{\mathrm{ATM}}
$$

The parameters $\hat{S}$ and $\hat{P}_{i}$ denote the peak amplitudes of the system waveform and the echo waveform, resp., whereas $s_{s}$ and $s_{p, i}$ are the corresponding widths of the respective Gaussian functions, expressed as standard deviations. The energy of the system waveform, $E_{S}$, is simply

$$
E_{S}=\int_{-\infty}^{\infty} S(t) \mathrm{d} t=\sqrt{2 \pi} \hat{S} s_{s}
$$

Separating the parameters of the reflecting surface from the other parameters of $\hat{P}_{i}$ leads to the introduction of the calibration constant $C_{\mathrm{CAL}}$ (Wagner et al., 2006; Briese et al., 2008):

$$
\sigma_{i}=C_{\mathrm{CAL}} R_{i}^{4} \hat{P}_{i} s_{p, i}
$$

with

$$
C_{\mathrm{CAL}}=\frac{4 \pi \beta_{t}^{2}}{\eta_{\mathrm{SYS}} \eta_{\mathrm{ATM}} D_{r}^{2} \hat{S} s_{s}}=\frac{4 \pi \sqrt{2 \pi} \beta_{t}^{2}}{\eta_{\mathrm{SYS}} \eta_{\mathrm{ATM}} D_{r}^{2} E_{S}}
$$

$C_{\mathrm{CAL}}$ can be calculated using naturally available (Briese et al., 2008; Lehner and Briese, 2010) or artificial reference targets (Kaasalainen et al., 2009) with known reflectivity. Its determination enables to derive $\sigma$ as radiometric quantity of the single scatterer independent of the parameters of the emitted laser pulse. However, $\sigma$ is influenced by the incidence angle $\vartheta$ of the laser beam to the scattering surface and the effective illuminated area $A$ of this scatterer. Thus, it is preferable to use the backscattering coefficient $\gamma$ instead of $\sigma$ (Wagner, 2010):

$$
\gamma=\frac{\sigma}{A \cos \vartheta}
$$

Since $A \cos \vartheta$ is the orthogonal projection of $A$ in the direction of the laser beam, $\gamma$ can be determined without regarding the local surface normal of the scatterer.

The parameters of the transmitted laser pulse are normally regarded as unknown (or known up to a constant factor since $S(t)$ is stored in a damped version) but constant quantities. This is also reflected in standards such as the ASPRS LAS 1.3 (LASer File Format Exchange, (ASPRS, 2011)) and the Riegl SDC file format (Riegl, 2011). Both do not represent the transmitted laser pulse. However, there has been empirical evidence that the transmitted laser pulse cannot be regarded as "constant enough" for proper radiometric calibration (cf. (Mallet, 2011; Bretar et al., 2009)). In (Wagner, 2010), a different version of the calibration constant is therefore formulated, without $\hat{S}$ in the denominator:

$$
\bar{C}_{\mathrm{CAL}}=\frac{4 \pi \beta_{t}^{2}}{\eta_{\mathrm{SYS}} \eta_{\mathrm{ATM}} D_{r}^{2} s_{s}} .
$$

\section{ERROR PROPAGATION DUE TO LASER PULSE VARIATIONS}

The observation of $S(t)$ and the determination of $\hat{S}$ (up to a constant factor) and $s_{s}$ allow us to study the influence of their variations on $C_{\mathrm{CAL}}$.

For this analysis, we first write the partial derivatives of $C_{C A L}$ w.r.t. $\hat{S}$ and $s_{s}$ :

$$
\begin{aligned}
& \frac{\partial C_{\mathrm{CAL}}}{\partial \hat{S}}=-\frac{1}{\hat{S}} C_{\mathrm{CAL}} \\
& \frac{\partial C_{\mathrm{CAL}}}{\partial s_{s}}=-\frac{1}{s_{s}} C_{\mathrm{CAL}}
\end{aligned}
$$

Following the law of error propagation, this yields for the variance $\varsigma_{C_{\mathrm{CAL}}}^{2}$ (Mikhail, 1976) ${ }^{1}$ :

$$
\begin{aligned}
& \varsigma_{C_{\mathrm{CAL}}}^{2}= \\
& \left(\frac{\partial C_{\mathrm{CAL}}}{\partial \hat{S}}, \frac{\partial C_{\mathrm{CAL}}}{\partial s_{s}}\right)\left(\begin{array}{cc}
\varsigma_{\hat{S}}^{2} & \rho \varsigma_{\hat{S}} \varsigma_{s_{s}} \\
\rho \varsigma_{\hat{S}} \varsigma_{s_{s}} & \varsigma_{s_{s}}^{2}
\end{array}\right)\left(\begin{array}{l}
\frac{\partial C_{\mathrm{CAL}}}{\partial \hat{S}} \\
\frac{\partial C_{\mathrm{CAL}}}{\partial s_{s}}
\end{array}\right)
\end{aligned}
$$

with $\rho$ as the correlation coefficient of $\hat{S}$ and $s_{s} . \varsigma_{\hat{S}}^{2}$ and $\varsigma_{s_{s}}^{2}$ denote

\footnotetext{
${ }^{1}$ The letter $\varsigma$ (sigma) is used to avoid confusion with the backscatter cross-section.
} 
the variances of $\hat{S}$ and $s_{s}$, resp. Reordering Equation (11) gives

$$
\begin{aligned}
\varsigma_{C_{\mathrm{CAL}}}^{2} & =\left(\frac{\partial C_{\mathrm{CAL}}}{\partial \hat{S}}\right)^{2} \varsigma_{\hat{S}}^{2}+\left(\frac{\partial C_{\mathrm{CAL}}}{\partial s_{s}}\right)^{2} \varsigma_{s_{s}}^{2} \\
& +2 \rho \frac{\partial C_{\mathrm{CAL}}}{\partial \hat{S}} \frac{\partial C_{\mathrm{CAL}}}{\partial s_{s}} \varsigma_{\hat{S}} \varsigma_{s_{s}}
\end{aligned}
$$

The relative deviation of $C_{\mathrm{CAL}}$ is therefore given by

$$
\frac{\varsigma_{C_{\mathrm{CAL}}}^{2}}{C_{\mathrm{CAL}}^{2}}=\frac{\varsigma_{\hat{S}}^{2}}{\hat{S}^{2}}+\frac{\varsigma_{s_{s}}^{2}}{s_{s}^{2}}+2 \rho \frac{\varsigma_{\hat{S}}}{\hat{S}} \frac{\varsigma_{s_{s}}}{s_{s}}
$$

When assuming positive correlation between $\hat{S}$ and $s_{s}$ (empirically justified by the data sets investigated in this study, see Section 5), a lower bound of the relative deviation of $C_{\mathrm{CAL}}$ is found by neglecting correlation $(\rho=0)$ :

$$
\frac{\varsigma_{C_{\mathrm{CAL}}}^{2}}{C_{\mathrm{CAL}}^{2}} \geq \frac{\varsigma_{\hat{S}}^{2}}{\hat{S}^{2}}+\frac{\varsigma_{s_{s}}^{2}}{s_{s}^{2}}
$$

Figure 1 shows the relation of these three relative deviations for different levels of correlation.
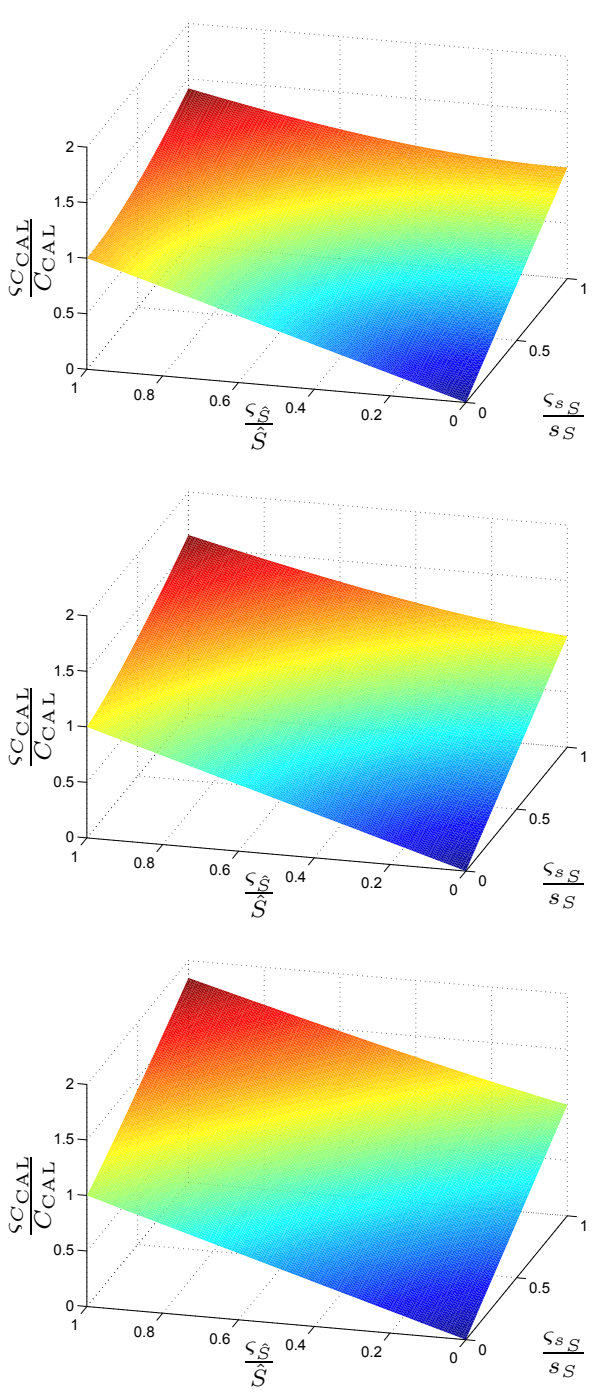

Figure 1: Error propagation of the relative deviations of the amplitude $(\hat{S})$ and the width $\left(s_{s}\right)$ resulting in the relative deviation of the calibration constant $C_{\mathrm{CAL}}$. Top: no correlation between $\hat{S}$ and $s_{s}$. Center: $\rho=0.3$. Bottom: $\rho=0.7$.

\section{DATA SETS}

Empirical analysis was carried out on the example of two fullwaveform ALS campaigns. The campaigns took place over the Schönbrunn area in Vienna, Austria on August 20, 2004 and May 4, 2005, resp. Both were operated with a Riegl LMS-Q560 instrument (Riegl, 2011). The first campaign consisted of eleven flight strips with 1.9 million to 2.9 million laser pulses per flight strip and 26.4 million laser pulses in total. The scanner was operated at a pulse repetition rate of $50 \mathrm{kHz}$.

The second campaign contained thirteen flight strips with 3.6 million to 5.0 million laser pulses per flight strip, resulting in approx. 53.1 million laser pulses all together. The pulse repetition rate was $100 \mathrm{kHz}$. The scan layout of both campaigns is shown in Figure 2.

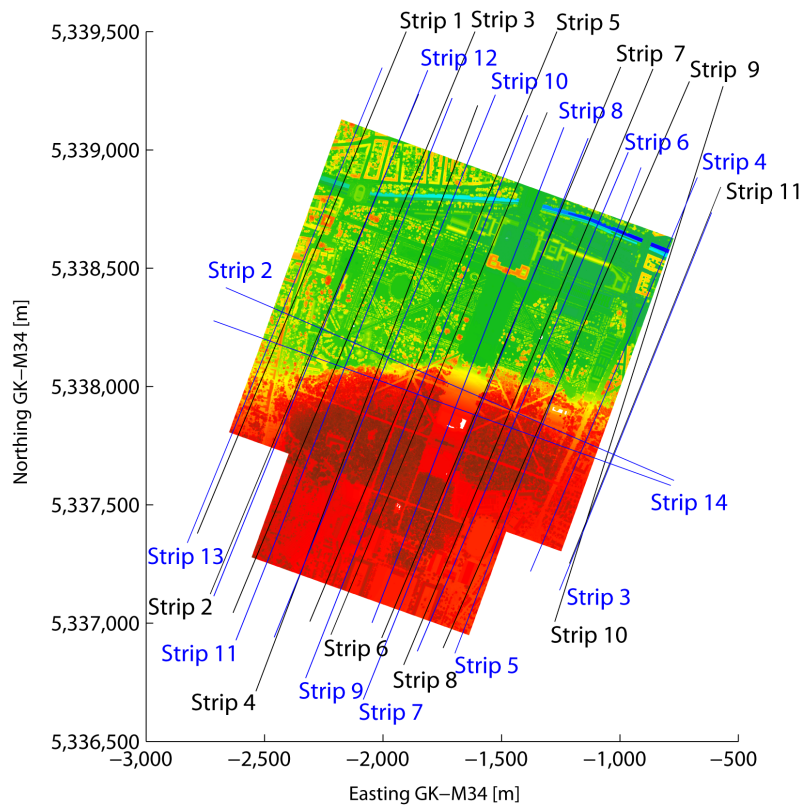

Figure 2: Digital surface model of the Schönbrunn area of Vienna overlaid with the flight trajectories of the two scanning campaigns of 2004 (black lines) and 2005 (blue lines).

The scan layouts of the two campaigns were nearly equivalent with the exception of the different pulse repetition rate and the two additional strips of the 2005 campaign (strips 2 and 14). The two campaigns form therefore an ideal test data set for investigating the validity of the calibration constant among different flight strips and different scanning campaigns regarding the variation of the emitted laser pulses.

\section{RESULTS}

For each recorded system waveform, its amplitude $\hat{S}$ and width $s_{s}$ were calculated using the Gaussian Decomposition algorithm suggested in (Wagner et al., 2006). We based our analysis on histograms and other statistics (minimum, maximum, mean, standard deviation and relative standard deviation), calculated per flight strip for $\hat{S}$ and $s_{s}$.

The 2004 campaign showed very similar distributions of $\hat{S}$ (given in Table 1 and Figure 3) with slightly different mean values per strip but very similar shapes. Only the flight strips 10 and 11 showed a noticeably higher skewness. The distributions of $s_{s}$ per flight strip were practically identical (see Table 2 and Figure 4). 


\begin{tabular}{crrrrr}
\hline Flight strip & min. & $\max$. & $\mu_{\hat{S}}$ & $\varsigma_{\hat{S}}$ & $\varsigma_{\hat{S}} / \mu_{\hat{S}}$ \\
\hline 1 & 193.4 & 264.3 & 226.0 & 7.3 & 0.032 \\
2 & 190.7 & 259.4 & 224.0 & 7.2 & 0.032 \\
3 & 188.3 & 256.8 & $\mathbf{2 2 2 . 8}$ & $\mathbf{7 . 1}$ & $\mathbf{0 . 0 3 2}$ \\
4 & 192.3 & 257.3 & 223.5 & 7.2 & 0.032 \\
5 & 192.6 & $\mathbf{2 5 5 . 7}$ & 223.2 & 7.2 & 0.032 \\
6 & 191.6 & 260.8 & 224.6 & 7.3 & 0.032 \\
7 & $\mathbf{1 8 5 . 9}$ & 260.8 & 225.5 & 7.3 & 0.032 \\
8 & 192.3 & 265.7 & 225.9 & 7.3 & 0.032 \\
9 & 193.8 & 262.8 & 226.9 & 7.4 & 0.033 \\
10 & 198.4 & 265.6 & $\mathbf{2 2 8 . 7}$ & $\mathbf{7 . 5}$ & $\mathbf{0 . 0 3 3}$ \\
11 & $\mathbf{1 9 9 . 8}$ & $\mathbf{2 6 8 . 1}$ & 228.4 & 7.5 & 0.033 \\
\hline Mean & - & - & 225.4 & 7.3 & 0.032 \\
\hline
\end{tabular}

Table 1: Statistics for the system waveform amplitudes $\hat{S}$ of the 2004 campaign (unit: DN). Bold figures denote the minima and maxima per category for the whole campaign, resp.

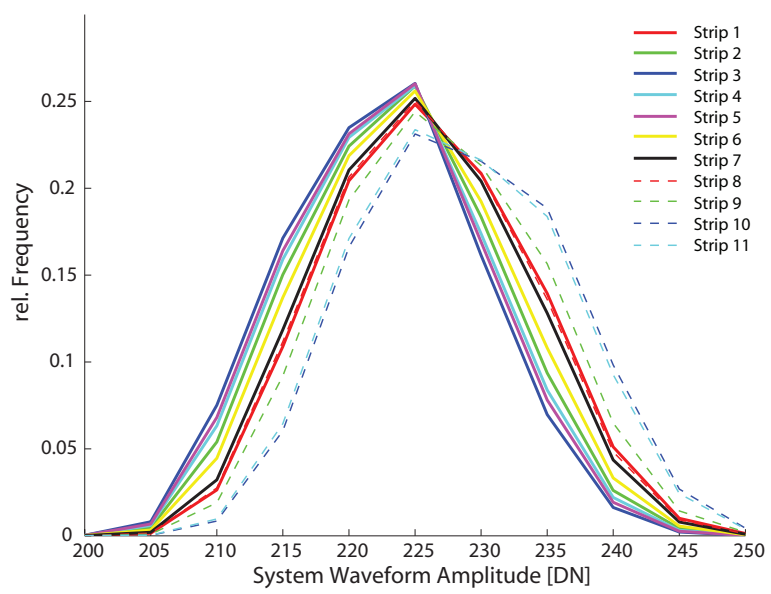

Figure 3: Histogram of the system waveform amplitudes $\hat{S}$ per flight strip in the 2004 campaign (bin size: 5).

\begin{tabular}{crrrrr}
\hline Flight strip & min. & max. & $\mu_{s_{s}}$ & $\varsigma_{s_{s}}$ & $\varsigma_{s_{s}} / \mu_{s_{s}}$ \\
\hline 1 & 1.804 & 1.963 & 1.861 & 0.0138 & 0.00745 \\
2 & 1.796 & 1.962 & 1.861 & 0.0139 & 0.00749 \\
3 & $\mathbf{1 . 8 0 6}$ & 1.966 & 1.861 & 0.0140 & 0.00750 \\
4 & 1.800 & 1.967 & 1.861 & $\mathbf{0 . 0 1 4 0}$ & $\mathbf{0 . 0 0 7 5 1}$ \\
5 & 1.800 & 1.968 & $\mathbf{1 . 8 6 1}$ & 0.0139 & 0.00745 \\
6 & 1.804 & 1.966 & 1.861 & 0.0139 & 0.00746 \\
7 & 1.805 & 1.963 & 1.861 & 0.0139 & 0.00748 \\
8 & 1.800 & $\mathbf{1 . 9 8 0}$ & 1.862 & 0.0139 & 0.00746 \\
9 & $\mathbf{1 . 7 9 5}$ & 1.965 & 1.862 & 0.0139 & 0.00746 \\
10 & 1.798 & 1.962 & 1.862 & $\mathbf{0 . 0 1 3 8}$ & $\mathbf{0 . 0 0 7 4 3}$ \\
11 & 1.803 & $\mathbf{1 . 9 6 1}$ & $\mathbf{1 . 8 6 2}$ & 0.0139 & 0.00749 \\
\hline Mean & - & - & 1.861 & 0.0139 & 0.00750 \\
\hline
\end{tabular}

Table 2: Statistics for the system waveform widths $s_{s}$ of the 2004 campaign (unit: $\mathrm{ns}$ ). Bold figures denote the minima and maxima per category for the whole campaign, resp.

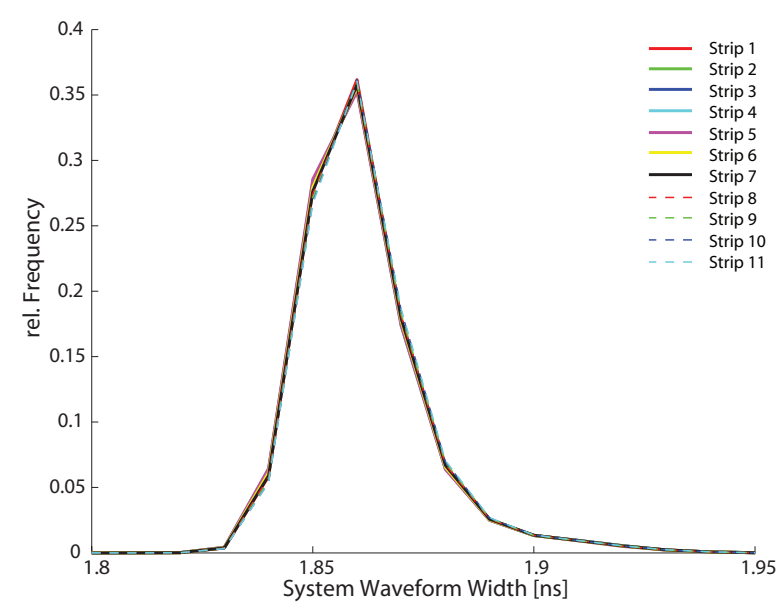

Figure 4: Histogram of the system waveform widths $s_{s}$ per flight strip in the 2004 campaign (bin size: 0.01).

The high similarity of the distributions of both $\hat{S}$ and $s_{s}$ in the 2004 campaign was not present in the other campaign. Especially the amplitudes showed a much higher variation which might be due to the higher pulse repetition rate. The mean values per flight strip ranged from 158.8 to 222.7 whereas the average value in the 2004 campaign was 225.4. However, the relative deviations per flight strip were comparable to those of the 2004 campaign, except for strip 2 where the relative deviation was more than three times higher. The two very small minimum amplitudes of strip 11 and 12 (1.2 and 3.6) are due to a erroneous recording, i.e. the digitizer was turned on although no laser pulse was emitted and only noise was recorded. Table 3 and Figure 5 contain the detailed figures and histograms.

\begin{tabular}{crrrrr}
\hline Flight strip & min. & max. & $\mu_{\hat{S}}$ & $\varsigma_{\hat{S}}$ & $\varsigma_{\hat{S}} / \mu_{\hat{S}}$ \\
\hline 2 & 126.8 & 242.8 & 161.2 & $\mathbf{1 9 . 5}$ & $\mathbf{0 . 1 2 1}$ \\
3 & 135.4 & $\mathbf{1 8 5 . 1}$ & $\mathbf{1 5 8 . 8}$ & 5.7 & 0.036 \\
4 & 143.3 & 189.0 & 165.1 & $\mathbf{5 . 5}$ & 0.033 \\
5 & 148.3 & 199.6 & 173.7 & 6.2 & 0.036 \\
6 & 160.9 & 224.2 & 193.7 & 7.5 & 0.038 \\
7 & 174.9 & 232.5 & 203.3 & 6.5 & 0.032 \\
8 & 179.6 & 242.3 & 210.3 & 7.1 & 0.034 \\
9 & 180.4 & 240.1 & 210.4 & 6.9 & 0.033 \\
10 & 183.2 & 244.5 & 214.9 & 7.0 & 0.033 \\
11 & 3.6 & 245.6 & 214.5 & 6.9 & 0.032 \\
12 & $\mathbf{1 . 2}$ & 249.9 & 219.6 & 7.1 & 0.032 \\
13 & $\mathbf{1 8 8 . 9}$ & 247.2 & 218.3 & 6.9 & $\mathbf{0 . 0 3 2}$ \\
14 & 188.1 & $\mathbf{2 5 2 . 8}$ & $\mathbf{2 2 2 . 7}$ & 7.2 & 0.032 \\
\hline Mean & - & - & 197.4 & 7.7 & 0.040 \\
\hline
\end{tabular}

Table 3: Statistics for the system waveform amplitudes $\hat{S}$ of the 2005 campaign (unit: DN). Bold figures denote the minima and maxima per category for the whole campaign, resp. 


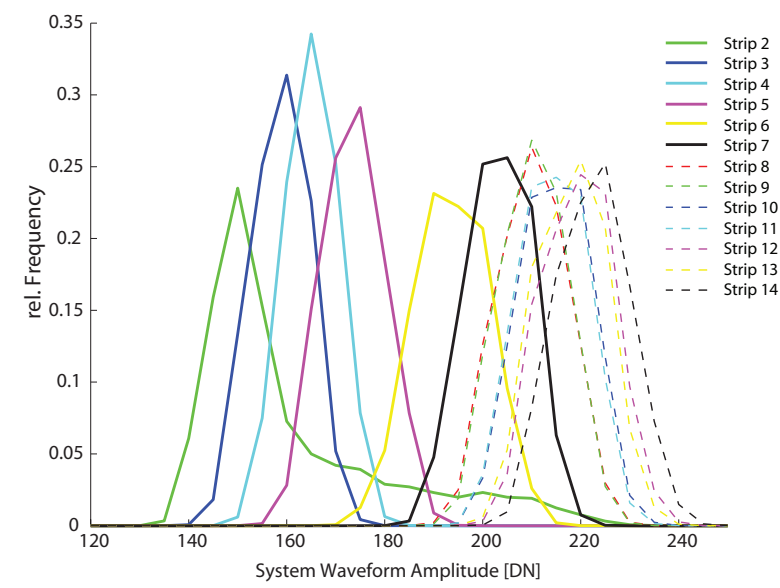

Figure 5: Histogram of the system waveform amplitudes $\hat{S}$ per flight strip of the 2005 campaign (bin size: 5 ).

The average of the pulse widths $s_{s}$ in the 2005 campaign differed about $1.5 \%$ from the average value of 2004 (1.835 ns vs. $1.861 \mathrm{~ns}$ ) with lower relative deviations. All flight strips showed very similar distributions, in contrast to the distributions of $\hat{S}$ (see Table 4 and Figure 5).

\begin{tabular}{crrrrr}
\hline Flight strip & min. & max. & $\mu_{s_{s}}$ & $\varsigma_{s_{s}}$ & $\varsigma_{s_{s}} / \mu_{s_{s}}$ \\
\hline 2 & 1.775 & 1.882 & 1.832 & $\mathbf{0 . 0 0 9 1 1}$ & $\mathbf{0 . 0 0 4 9 7}$ \\
3 & 1.777 & 1.877 & $\mathbf{1 . 8 3 2}$ & 0.00894 & 0.00488 \\
4 & 1.767 & 1.880 & 1.835 & 0.00871 & 0.00475 \\
5 & 1.775 & 1.881 & 1.835 & 0.00840 & 0.00458 \\
6 & 1.783 & $\mathbf{1 . 8 7 3}$ & 1.834 & 0.00786 & 0.00428 \\
7 & 1.777 & 1.876 & 1.834 & 0.00770 & 0.00420 \\
8 & 1.781 & 1.877 & 1.835 & 0.00763 & 0.00416 \\
9 & $\mathbf{1 . 7 8 6}$ & 1.874 & 1.836 & 0.00763 & 0.00416 \\
10 & 1.774 & 1.875 & 1.836 & 0.00761 & 0.00414 \\
11 & $\mathbf{0 . 3 6 3}$ & 1.882 & 1.837 & 0.00765 & 0.00416 \\
12 & 1.776 & $\mathbf{2 . 2 0 7}$ & 1.837 & 0.00756 & 0.00410 \\
13 & 1.776 & 1.875 & 1.837 & 0.00756 & 0.00412 \\
14 & 1.782 & 1.879 & $\mathbf{1 . 8 3 8}$ & $\mathbf{0 . 0 0 7 4 9}$ & $\mathbf{0 . 0 0 4 0 8}$ \\
\hline Mean & - & - & 1.835 & 0.00800 & 0.00440 \\
\hline
\end{tabular}

Table 4: Statistics for the system waveform widths $s_{s}$ of the 2005 campaign (unit: ns). Bold figures denote the minima and maxima per category for the whole campaign, resp.

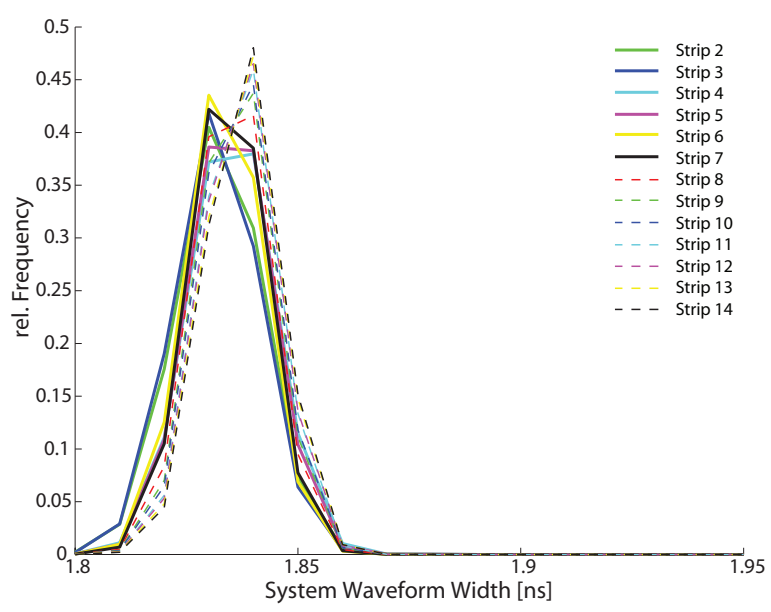

Table 5: Histogram of the system waveform widths $s_{s}$ per flight strip of the 2005 campaign (bin size: 0.01 ).
Besides the statistics of amplitudes and widths of the emitted laser pulses, also their correlation coefficients $\rho$ were calculated per flight strip. Their values varied from 0.23 to 0.24 in the 2004 campaign and from 0.02 to 0.18 in the campaign of 2005 .

These results enable us to calculate upper bounds of their influence on the calibration constant by evaluating Equation (12). Taking the respective maximal values in Tables $1-4$, we see that the relative deviation of $C_{\mathrm{CAL}}$ is

$$
\begin{aligned}
\frac{\varsigma_{C_{\mathrm{CAL}}}}{C_{\mathrm{CAL}}} & \leq \sqrt{0.033^{2}+0.00751^{2}+2 \cdot 0.24 \cdot 0.033 \cdot 0.00751} \\
& =0.0356
\end{aligned}
$$

within the single flight strips of 2004,

$$
\begin{aligned}
\frac{\varsigma_{C_{\mathrm{CAL}}}}{C_{\mathrm{CAL}}} & =\sqrt{0.121^{2}+0.00497^{2}+2 \cdot 0.14 \cdot 0.121 \cdot 0.00497} \\
& =0.1218
\end{aligned}
$$

for strip 2 of the 2005 campaign (where $\rho=0.14$ ) and

$$
\begin{aligned}
\frac{\varsigma_{C A L}}{C_{\mathrm{CAL}}} & \leq \sqrt{0.038^{2}+0.00488^{2}+2 \cdot 0.18 \cdot 0.038 \cdot 0.00488} \\
& =0.0392
\end{aligned}
$$

for all other single strips of this campaign. The variation of $\hat{S}$ can be regarded as main influence quantity for the variation of $C_{\mathrm{CAL}}$ so that $s_{s}$ and the correlation between these two parameters can be neglected.

Our results also apply if an other deconvolution technique than Gaussian Decomposition is performed. In this case, the righthand side of Equation (6) is taken into account so that the relative deviation of $C_{\mathrm{CAL}}$ is only dependent on the relative deviation of system waveform energy, $\varsigma_{E_{S}} / E_{S}$. Their distribution was also analysed but not separately listed here. It showed a distribution similar to the one of the system waveforms' amplitudes as was expected due to the small variations of the widths $s_{s}$.

\section{DISCUSSION AND OUTLOOK}

In this study, the variations of emitted laser pulses were evaluated on behalf of two flight campaigns of 2004 and 2005, one operated with a pulse repetition rate of $50 \mathrm{kHz}$, the other with $100 \mathrm{kHz}$. The results of the 2004 campaign showed similar distributions for all flight strips whereas the 2005 data set is characterized by narrow distributions within the single flight strips but significantly different behaviour between this strips. The relative deviation of $C_{\mathrm{CAL}}$ was around $3-4 \%$ per flight strip in our data sets. However, it was a lot higher within the whole 2005 ALS campaign as well as between the 2004 and the 2005 campaign although the same instrument was in use. The mean value for the system waveform amplitudes amounted to 225.4 with a relative deviation of $3.2 \%$ in the 2004 campaign and to 197.4 in the 2005 campaign (relative deviation: $4.0 \%$ ). The mean of the system waveform widths resulted to $1.861 \mathrm{~ns}$ and $1.835 \mathrm{~ns}$, resp. In both cases, their relative deviations were smaller than $1 \%$.

The current state of the art within the process of radiometric calibration is the determination of one singular calibration constant per flight campaign, based on artificial or naturally available targets. This study demonstrates the presence of laser pulse variations (amplitude and pulse width) within a strip as well as in the whole flight campaign and estimates their influence on the determination of the calibration constant. Within one strip, a narrow variation can be observed whereas especially in flight campaign 2005, a high variation between the strips occurred. For an advanced radiometric calibration, one can focus on the calculation 
of an individual calibration constant per strip. This would allow to reduce the variation of the radiometric calibration between the strips. The determination of a shot-based calibration constant is not feasible. However, in order to consider the individual variation of the laser pulse, either

- the individual amplitudes and pulse widths can be considered as additional variables in the whole radiometric calibration process (cf. (Wagner, 2010)) or

- by using echo parameters normalized by the parameters of the individual laser pulses instead of the original echo parameters in the mathematical framework of radiometric calibration. This would enable to consider the pulse variations without increasing storage space and processing time. Furthermore, it is compatible with currently available file format standards.

Future research and further practical investigations will show which of these methods is more practicable.

\section{ACKNOWLEDGEMENTS}

We would like to thank Schloß Schönbrunn Kultur- und Betriebsges.m.b.H and Milan-Flug GmbH for their support in the data acquisition campaigns. Furthermore, we want to thank Norbert Pfeifer and Helmut Kager (both IPF) for the fruitful discussions.

The first author has been supported by a Karl Neumaier PhD scholarship.

The Ludwig Boltzmann Institute for Archaeological Prospection and Virtual Archaeology is based on an international cooperation of the Ludwig Boltzmann Gesellschaft (Austria), the University of Vienna (Austria), the Vienna University of Technology (Austria), the Austrian Central Institute for Meteorology and Geodynamics, the office of the provincial government of Lower Austria, RGZM (Roman-Germanic Central Museum Mainz, Germany), RA (Swedish National Heritage Board), VISTA (Visual and Spatial Technology Centre, University of Birmingham, UK) and NIKU (Norwegian Institute for Cultural Heritage Research).

\section{References}

ASPRS, 2011. LAS file format exchange activities. http://www.asprs.org/Standards/ LASer-LAS-File-Format-Exchange-Activities . html. Homepage of ASPRS LAS file format, accessed: April 2011.

Bretar, F., Chauve, A., Bailly, J.-S., Mallet, C. and Jacome, A., 2009. Terrain surfaces and 3-d landcover classification from small footprint full-waveform lidar data: Application to badlands. Hydrology and Earth System Sciences 13(8), pp. 1531-1545.

Briese, C., Höfle, B., Lehner, H., Wagner, W. and Pfenningbauer, M., 2008. Calibration of full-waveform airborne laser scanning data for object classification. In: SPIE: Laser Radar Technology and Applications XIII.

Höfle, B. and Pfeifer, N., 2007. Correction of laser scanning intensity data: Data and model-driven approaches. ISPRS Journal of Photogrammetry and Remote Sensing 62(6), pp. 415-433.
Hofton, M., Minster, J. and Blair, J., 2000. Decomposition of laser altimeter waveforms. IEEE Transactions on Geoscience and Remote Sensing 38, pp. 1989-1996.

Jelalian, A. V., 1992. Laser Radar Systems. Artech House, Boston.

Jutzi, B. and Stilla, U., 2006. Range determination with waveform recording laser systems using a wiener filter. ISPRS Journal of Photogrammetry and Remote Sensing 61(1), pp. 95-107.

Kaasalainen, S., Hyyppä, H., Kukko, A., Litkey, P., Ahokas, E., Hyyppä, J., Lehner, H., Jaakkola, A., Suomalainen, J., Akujarvi, A., Kaasalainen, M. and Pyysalo, U., 2009. Radiometric calibration of lidar intensity with commercially available reference targets. IEEE Transactions on Geoscience and Remote Sensing 47(2), pp. 588-598.

Lehner, H. and Briese, C., 2010. Radiometric calibration of full-waveform airborne laser scanning data based on natural surfaces. In: ISPRS Technical Commission VII Symposium 2010: 100 Years ISPRS - Advancing Remote Sensing Science. International Archives of the Photogrammetry, Remote Sensing and Spatial Information Sciences 38 (Part 7B), Vienna, Austria, pp. 360365.

Mallet, C., 2011. Analyse de données lidar à Retour d'Onde Complète pour la classification en milieu urbain. PhD thesis, Télécom ParisTech, École Doctorale d'Informatique, Télécommunications et Électronique de Paris, Paris, France.

Mallet, C. and Bretar, F., 2009. Full-waveform topographic lidar: State-of-the-art. ISPRS Journal of Photogrammetry and Remote Sensing 64(1), pp. 1-16.

Mikhail, E. M., 1976. Observations And Least Squares. IEP-A Dun-Donnelley, New York.

Parrish, C. E. and Nowak, R. D., 2009. Improved Approach to Lidar Airport Obstruction Surveying Using Full-Waveform Data. Journal of Engineering Surveying 135(2), pp. 72-82.

Riegl, 2011. www.riegl.com. Homepage of the company RIEGL Laser Measurement Systems GmbH, accessed: April 2011.

Roncat, A., Bergauer, G. and Pfeifer, N., 2011. B-Spline Deconvolution for Differential Target Cross-Section Determination in Full-Waveform Laser Scanner Data. ISPRS Journal of Photogrammetry and Remote Sensing 66(4), pp. 418-428.

Wagner, W., 2010. Radiometric calibration of smallfootprint full-waveform airborne laser scanner measurements: Basic physical concepts. ISPRS Journal of Photogrammetry and Remote Sensing 65(6 (ISPRS Centenary Celebration Issue )), pp. 505-513.

Wagner, W., Ullrich, A., Ducic, V., Melzer, T. and Studnicka, N., 2006. Gaussian decomposition and calibration of a novel small-footprint full-waveform digitising airborne laser scanner. ISPRS Journal of Photogrammetry and Remote Sensing 60(2), pp. 100-112. 